\title{
Prevalence of Anti-Toxoplasma gondii Antibody in Hunter-Killed Wild Boars, Sus scrofa leucomystax, on Amakusa Island, Kumamoto Prefecture, Japan
}

\author{
Takashi SHIIBASHI ${ }^{1)}$, Koichiro NARASAKI ${ }^{2)}$, Motonobu YOSHIDA ${ }^{1)}$ and Sadao NOGAMI ${ }^{1)}$ \\ ${ }^{1)}$ Department of Veterinary Medicine, College of Bioresource Sciences, Nihon University, Kameino 1866, Fujisawa, Kanagawa $252-8510$ \\ and ${ }^{2)}$ Narasaki Veterinary Hospital, Hirose 133-3, Hondo-machi, Hondo-shi, Kumamoto 863-0001, Japan
}

(Received 23 May 2003/Accepted 13 November 2003)

ABSTRACT. The prevalence of Toxoplasma gondii was surveyed in wild boars (Sus scrofa leucomystax) and domiciled cats obtained in various areas of Amakusa Island, Kumamoto Prefecture, Japan. The antibody titers against T. gondii were measured with a latex agglutination test. Among specimens taken from 90 wild boars, $1(1.1 \%)$ was positive and $3(3.3 \%)$ were doubtfully positive. Among the specimens from 50 cats, none were positive and $1(3.3 \%)$ was doubtfully positive. These results suggest that the wild boars and cats on Amakusa Island have quite low prevalence of the T. gondii infection. Continuous surveys will be needed to monitor the prevalence of Toxoplasmosis and other zoonoses in game animals.

KEY WORDs: feline, Toxoplasma gondii, wild boar.

J. Vet. Med. Sci. 66(3): 327-328, 2004

Fetal mortality in pregnant women makes toxoplasmosis one of Japan's most serious public health risks. The causative agent, Toxoplasma gondii, is dependent on Felidae as its final host, and the infection has also been reported in various kinds of wildlife, including wild boars (Sus scrofa leucomystax) acting as intermediate hosts $[1,2,4]$.

Authorities in Japan have recently been forced to exterminate large numbers of wild boars due to their harmful effects and increasing numbers nearby human communities. Hunters usually scrap the wild boars they kill, but in some cases they and others consume the meat of the game. This behavior poses a risk to public health, as wild boars and other game animals often have zoonotic infectious diseases [4]. As the wild boar in Japan has been noted to have a high prevalence of anti- $T$. gondii antibody $[9,12]$, those that are hunted and consumed may comprise one source of toxoplasmosis in humans. In this study we investigated the prevalence of anti-T. gondii antibody in wild boar killed by hunters, in order to confirm the accompanying public health risk on Amakusa, an island located in the west of Kumamoto Prefecture, Japan. We also surveyed the antibody in cats domiciled in the same area, a likely source of $T$. gondii infection in wild animals.

Samplings were carried out in various areas of Amakusa from July 2000 to August 2001. Strips of quantitative blood collection filter paper (Advantec, Japan) were used to collect the blood samples. Although this method cannot properly measure antibody titer, it is useful to collect samples without syringe and needle. Moreover, this technique is well established and has been applied in field surveys, particularly in tropical areas [3,7]. A previous study has established that storage of the anti-T. gondii antibody on silica gel successfully preserves the activity of the antibody for an extended period [10].

Hunters in the field collected a total of 90 blood samples from wild boars they had just killed, as well as data on the individual animals, namely, the date and area of the killing, the sex, the estimated age and weight, the pregnancy status, and autopsy findings. The sampled filter papers were transferred from the field individually in securely sealed plastic bags with silica gel at room temperature under a lightshielded condition. Once in the laboratory, they were stored at $-30^{\circ} \mathrm{C}$ until elution. In a similar fashion, 50 blood samples were obtained from domiciled cats visiting an animal hospital located in the central region on Amakusa Island. The cats ranged in age between 1 and 13 years old. The number of cats aged 1-4, 5-9 and 10-13 years old were 24, 22 and 4, respectively. The cats were raised either freely (both indoors and outdoors) or predominantly outdoors. Elution was carried out as described previously [10], and the eluate was regarded as equivalent to a 16-fold dilution of serum [10]. The antibody titers against $T$. gondii were measured with a commercially available latex agglutination test kit, TOXOCHECK-MT 'Eiken' (Eiken-Kagaku, Japan). For the screening test, $25-\mu l$ of the eluate was diluted with an equal volume of buffered saline and serial 2-fold dilutions were made from 1:32 to 1:64. Further dilutions and tests were carried out to determine the actual antibody titer when the titer was diluted to concentrations weaker than $1: 64$. Antibody titers of 1:32 and 1:64 or above were regarded as doubtfully positive and positive, respectively [13].

Among the specimens sampled from 90 wild boars, 1 $(1.1 \%)$ was positive and $3(3.3 \%)$ were doubtfully positive (Table 1). Among the specimens from 50 cats, none were positive and 1 (3.3\%) was doubtfully positive (Table 2 ).

The prevalence of toxoplasmosis in wild boars on Amakusa Island (1.1\%) was apparently lower than the prevalences recorded in the Kobe district $(52.9 \%)$ and on Iriomote Island (5.6\%), Japan [9, 12]. This difference compelled us to examine the prevalence in cats, the final host of $T$. gondii and a possible source of toxoplasmosis 
Table 1. Distribution of anti-Toxoplasma gondii antibody titers ${ }^{\text {a) }}$ in wild boar, Sus scrofa leucomystax, on Amakusa Island, Japan

\begin{tabular}{lcccccc}
\hline \multirow{2}{*}{$\begin{array}{l}\text { Sex and body } \\
\text { weight }(\mathrm{kg})\end{array}$} & $\begin{array}{c}\text { No. } \\
\text { examined }\end{array}$ & $\leq 1: 16$ & $1: 32$ & $1: 64$ & $>1: 64$ & Positive no. \\
\cline { 3 - 7 } & & & & & & \\
\hline Females & 10 & 10 & 0 & 0 & 0 & 0 \\
$<30$ & 11 & 11 & 0 & 0 & 0 & 0 \\
$30-50$ & 13 & 13 & 0 & 0 & 0 & 0 \\
$>50$ & 34 & 34 & 0 & 0 & 0 & 0 \\
Sub-total & & & & & & 0 \\
Males & 13 & 12 & 1 & 0 & 0 & 0 \\
$<30$ & 20 & 19 & 1 & 0 & 0 & 0 \\
$30-50$ & 22 & 20 & 1 & 1 & 0 & 1 \\
$>50$ & 55 & 51 & 3 & 1 & 0 & 1 \\
Sub-total & 1 & 1 & 0 & 0 & 0 & 0 \\
No data & 1 & 1 & 0 & 0 & 0 & 0 \\
Sub-total & & & & & & \\
Total & 90 & 86 & 3 & 1 & 0 & 1 \\
\hline
\end{tabular}

a) Titer 1:64 or above was regarded as positive.

Table 2. Distribution of anti-Toxoplasma gondii antibody titers ${ }^{\mathrm{a})}$ in cats on Amakusa Island, Japan

\begin{tabular}{lccccc}
\hline & $\begin{array}{c}\text { No. examined } \\
\text { (Spayed or } \\
\text { Sex }\end{array}$ & \multicolumn{4}{c}{ Anti-T. gondii antibody titer ${ }^{\mathrm{a}}$ ) } \\
\cline { 3 - 6 } & castrated) & $\leq 1: 16$ & $1: 32$ & $>1: 64$ & Positive no. \\
\hline Females & $18(10)$ & 17 & 1 & 0 & 0 \\
Males & $32(20)$ & 32 & 0 & 0 & 0 \\
Total & 50 & 49 & 1 & 0 & 0 \\
\hline
\end{tabular}

a) Titer 1:64 or above was regarded as positive.

contamination. As reported earlier in a survey conducted in Kumamoto Prefecture in 1998 [11], no seropositive domiciled cat were observed on Amakusa Island. Thus, the positive rates in wild boars and domiciled cats on Amakusa Island seemed to be correlated with each other. Since there are no wild Felidae on Amakusa Island, it was speculated that domiciled cats on Amakusa Island had originally been considerably free from $T$. gondii and the introduction of cats or intermediate hosts infected with $T$. gondii has been the exclusive invasion route to the island. While the results of this study suggest that the animals of Amakusa have quite a low prevalence of the $T$. gondii infection, they in no way predict the public health risks posed by wild boars in the future, as there are few safeguards to stop cats or intermediate hosts infected with $T$. gondii from coming to the island.

Japanese people often consume raw meat from game animals such as wild boar and deer, and this exposes them to the risk of infection from parasitic zoonoses. Indeed, there have been several documented cases of paragonimiasis among patients who consumed raw or undercooked meat of wild boar in Japan [6, 8]. Moreover, one study reported a high prevalence of the $T$. gondii antibody in slaughterhouse workers [5]. Since hunters usually scrap the game animals they kill, contact with the animal carcasses may be another important route of $T$. gondii infection besides the consumption of the raw meat. Hunters should be made aware of the T. gondii infection and advised to take due precautions when scrapping their game. For these reasons, it will be necessary to continuously monitor the prevalence of both toxoplasmosis and zoonotic diseases originating from other causative agents in wild boars.

This work was partly supported by a grant from the Ministry of Education, Culture, Sports, Science and Technology of Japan (14560273).

\section{REFERENCES}

1. Dreesen, D. W. 1990. J. Am. Vet. Med. Assoc. 196: 274-276.

2. Dubey, J. P. and Beattie, C. P. 1988. Toxoplasmosis of Animals and Man, CRC Press, Florida.

3. Evengard, B., Linder, E. and Lundbergh, P. 1997. Ann. Trop. Med. Parasitol. 82: 295-303.

4. Gibbs, E. P. 1997. Rev. Sci. Tech. 16: 594-598.

5. Horio, M., Nakamura, K. and Shimada, M.2001. J. UOEH. 23: 233-243.

6. Hatsushika, R. 2000. Kawasaki Med. J. 26: 65-82 (in Japanese, with English abstract).

7. Kanamura, H. Y., Dias, L. C., da Silva, R. M., Glasser, C. M., Patucci, R.M. and Vellosa, S. A. and Antunes, J. L. 1998. Rev. Inst. Med. Trop. Sao Paulo. 40: 85-91.

8. Kawanaka, M., Sugiyama, H. and Kato, K. 1999. Jpn. J. Trop. Med. Hyg. 27: 138.

9. Murata, K. 1988. J. Jpn. Vet. Med. Assoc. 41: 811-813 (in Japanese, with English abstract).

10. Nogami, S., Kamata, H., Maruyama, S., Furuya, H. and Inoue, I. 1992. Res. Vet. Sci. 52: 387-388.

11. Nogami, S., Moritomo, T., Kamata, H., Tamura, Y., Sakai, T., Nakagaki, K. and Motoyoshi, S. 1998. J. Vet. Med. Sci. 60: 1001-1004.

12. Nogami, S., Tabata, A., Moritomo, T. and Hayashi, Y. 1999. Vet. Res. Commun. 23: 211-214.

13. Tsubota, N., Hiraoka, K., Sawada,Y., Watanabe, T. and Ohshima, S. 1977. Jpn. J. Parasitol. 26: 286-290. 